\title{
Triggerare
}

\section{Simona Cresti}

PUBBLICATO: 31 MARZO 2019

\begin{abstract}
Quesito:
Un lettore ci segnala il verbo triggerare nel significato, diffusissimo sul web, di 'provocare una reazione di rabbia in qualcuno': è un neologismo?
\end{abstract}

\section{Triggerare}

Le domande che arrivano giornalmente alla redazione dell'Accademia costituiscono un osservatorio prezioso sulla percezione della lingua da parte dei parlanti. In questo caso, per esempio, una segnalazione ci informa che il verbo triggerare, non (o forse non ancora) accolto da nessuno dei principali dizionari italiani, si è diffuso - per lo meno in certi contesti - con una viralità tale da essere percepito da qualcuno come un neologismo. Dal punto di vista morfologico, triggerare è un ibridismo, vale a dire una parola formata dalla combinazione di elementi di lingue diverse: nel nostro caso, può essere interpretata come il risultato dell'apposizione del suffisso italiano -are alla base inglese trigger 'grilletto, innesco', oppure come l'adattamento del verbo inglese (to) trigger 'innescare'. Il sostantivo trigger, come vedremo, vive in italiano da diversi anni come prestito non adattato: la familiarità di molti parlanti con esso e, più in generale, con la lingua inglese lascia spazio a pochi dubbi sulla pronuncia del nostro verbo, che sarà "triggherare" con la g velare (ossia "dura").

Come vedremo, il verbo (to) trigger, in inglese, ha un uso più ampio e solo in parte sovrapponibile a quello del corrispondente italiano che ha suscitato la domanda giunta alla nostra redazione. Nel significato che il nostro lettore ci segnala triggerare appare legato agli ambiti d'uso del web, dei social network, dei forum, in cui può essere usato per descrivere l'azione del "pungere nel vivo" e del provocare in qualcuno una reazione di rabbia, irritazione, stizza. Diciamo, anche relativamente a questi contesti, che "puó" essere usato: persino in ambiti relativamente ristretti, infatti, gli impieghi del verbo appaiono poco omogenei.

\section{Qual è il significato della parola che ci segnalano? Qualche esempio}

Possiamo comprendere meglio il significato di triggerare se ne osserviamo l'uso in contesti reali. Tendenzialmente lo troviamo impiegato in costrutti transitivi come "triggerare qualcuno". In questo caso il suo significato è molto spesso quello segnalato dal nostro lettore ('far arrabbiare', o anche 'pungere nel vivo'). L'azione può essere compiuta da una persona, magari con una battuta provocatoria:

Prima di tutto scusa se ti ho triggerato con l'affermazione sul segnalibro di chrollo, non sto neanche a spiegarti cosa intendevo con quella frase sennò qui si passa gli anni a discutere [...] (intervento sul forum IGNItalia, 4/9/2017).

Come ti triggero il leghista contro Salvini (intervento sul forum termometropolitico.it, $2 \mathrm{2} / 2 / 2019$ ).

o provocata da una cosa:

Re: Esplosioni di rabbia, vi capitano?

Mi capitavano da giovane, probabilmente se fossi nato negli USA sarei finito sui giornali. La cosa che mi "triggerava" erano i momenti in cui venivo offeso, preso per il culo o considerato meno degli altri per cose su cui non avevo nessun controllo tipo framecellismo, statura e problemi di salute. Mi sfogavo bullizzando pesantemente gli omega della scuola, alla fine "shit always flows downhill" (intervento sul forum brutti.red, 3/3/2019).

Triggerati significa proprio quello, 'innescati', rispetto a qualche cosa, in genere rispetto a una tematica. Si è triggerati quando qualcuno fa quella affermazione per cui voi voi scattate lancia in resta contro quella persona. È un trigger qualcuno che mi parla di vaccini e dice "ah no perché i vaccini fanno venire l'autismo" - nel mio caso, eh! i vostri possono essere totalmente differenti -, è un trigger la stupidità, è un trigger il condividere delle fake news palesi... ci sono delle cose che mi fanno scattare (trascrizione nostra del video di Matteo Flora, E tu? che cosa ti triggera?, Youtube, 19/2/2019).

Come giustamente suggerisce chi parla nell'ultimo esempio, è ragionevole presumere che triggerare finisca per 
diventare un sinonimo di provocare o far arrabbiare perché, si sottintende, chi compie l'azione innesca reazioni di rabbia, irritazione, stizza. Se ciò che provoca è una cosa (un oggetto, unesperienza, un rumore, ecc.) il suo ruolo è descritto come quello di un "trigger emozionale" o "emotivo". Troviamo triggerare, infatti, usato - più raramente - anche in costrutti del tipo "triggerare qualcosa", dove appunto il "qualcosa" è tendenzialmente una reazione negativa del comportamento o dell'umore.

Vuoi triggerare una reazione compulsiva? Ma secondo te ci casco? :D :D :D (intervento sul forum termometropolitico.it, $22 / 3 / 2016)$.

È scontato che un insulto a sfondo razziale, religioso o sessista possa "triggerare" una reazione (Raffaele Alberto Ventura, Il politicamente da correggere, sulla rivista web Il maschile del Sole24Ore, 28/II/2016).

"Fanculo Kyle: non so come aiutarti a staccarti di dosso l'etichetta di morto di figa! rispose a quel punto, cercando di triggerare una reazione alterata dell'altro, o imbarazzarlo e distoglierlo così dalla sua debacle (stralcio di racconto pubblicato sul forum owari.forumfree.it, $14 / 9 / 2017$ ).

Il fatto che sul web non ci sia traccia di espressioni come "triggerare/triggera + gioia, felicità, allegria" consolida l'impressione che, per la maggior parte dei suoi impieghi, descrivere qualcuno come triggerato equivalga a dirlo innervosito, urtato, arrabbiato.

Vale la pena notare come in costruzioni passive o in funzione aggettivale il significato di essere triggerato può spingersi a indicare non solo l'essere stato indotto ad arrabbiarsi (un evento puntuale), ma anche il trovarsi in una condizione di arrabbiatura, irritabilità, inquietudine (uno stato, una situazione):

Eppure ti sentirai triggerato da questo meme e vorrai intervenire per confutarlo (post sulla pagina Facebook "Il maschio beta", $16 / 4 / 2017)$.

Cerco di coinvolgere comunque più persone possibili (anche se non ho più fantasia a dire il vero) ma mi esprimo come se fossimo tutti amici (tanto da, purtroppo, abusare di parolacce quando sono particolarmente triggerato, sono una brutta persona) (post sulla pagina Facebook "Soft-boiled", r7/9/2016).

Continuando ad osservare gli esempi tratti dal web, notiamo come in molti casi l'uso di triggerare si modelli su quello di certi suoi sinonimi intransitivi pronominali. Dal punto di vista grammaticale, in questi casi il suo comportamento è del tutto simile a quello di irritare. Nelle costruzioni attive è un normale verbo transitivo; quando è seguito dal pronome clitico -si, invece, non assume la sfumatura riflessiva ('irritare sé stesso', 'triggerare sé stesso'), ma una sfumatura di vantaggio ('sperimentare una reazione di irritazione/rabbia'):

Mi triggero quando ti trattano male (tweet del $2 \mathrm{I} / \mathrm{II} / 2018$ ).

Quando un comico inizia a scimmiottare il classoco [sic] tono della white girl di Los Angeles mi triggero. Che palle (intervento sul forum IGNItalia del $2 \mathrm{r} / \mathrm{II} / 20 \mathrm{I} 8$ ).

Basta che non osate mettere quel rifiuto di astolfo che mi triggero male (intervento sul forum serialclick.it, 20I8).

Similmente, dietro all'uso assoluto di triggerare ("qualcuno triggera", anch'esso possibile) è facile notare l'attrazione del giovanile sclerare: in questi casi il nostro verbo significa, come il suo modello, "uscire fuori di testa".

[...] ho triggerato male per via della stanchezza (intervento sul forum gamesvillage.it, $7 / 10 / 2017$ ).

Come nell'esempio precedente, anche in questo ricorre l'accostamento con l'avverbio di modo male. In questi casi male sembra usato in senso intensivo, e dunque rafforzare un'espressione che già si riferisce a qualcosa di negativo. D'altra parte, in contesti colloquiali, giovanili e sul web non è raro imbattersi in usi di male paragonabili a questo: basti pensare alla sua associazione al verbo "morire" in espressioni di malaugurio (9.700 risultati per "muori male"), o in iperboli ironiche ( 7800 per "muoio male"). Nei nostri esempi, dunque, "mi triggero male" equivale allora a qualcosa come "mi arrabbio di brutto", e "ho triggerato male" a "ho sclerato di brutto".

Il confronto con l'accostamento all'avverbio bene (a dire la verità piuttosto raro) appare interessante. In alcuni casi bene, come il male degli esempi precedenti, si riferisce soltanto alla modalità in cui l'azione è svolta. Nel 
contesto che segue, per esempio, qualcuno non è stato fatto arrabbiare abbastanza:

Penso tu debba riprovare a scrivergli fino a che non ti augura deportazione in Corea: non l'hai triggerato bene. \#GrandeGiornalismo (tweet del 3/4/29017).

In altri casi, invece, bene è in grado di alterare il significato del nostro verbo. Nel prossimo esempio triggerare sembra avere il significato, diremmo "neutro", di 'attivare', e quindi descrivere, in associazione con bene, una reazione di commozione e trasporto; in associazione con male, una reazione di stizza.

Ho un'amica (IRL) a cui ho dedicato dei capitoli e tipo lei "Mi commuovo quando li scrivi". Cosi la triggero bene anche adesso lol- [...]. Ora la triggero male: HO PRESO 8 IN LATINO SENZA STUDIARE AHAHAHAHAHAHAHAHAH (intervento sulla community wattpad.com).

Per quanto l'associazione con bene sia presente in usi isolati, non rappresentativi di una porzione significativa di dati, l'ultimo esempio ci spinge a riflettere ancora sul significato del verbo. Sul web, infatti, e in particolare ancora sui forum e sui social network, troviamo testimonianze di untriggerare usato con un significato sensibilmente diverso da (a volte quasi opposto a) quello segnalato dal lettore. In questi casi ad essere attivate non sono rabbia e irritazione, ma reazioni variamente piacevoli.

[...] il suono è una vibrazione e le vibrazioni sono energia,quindi la musica anche per me funziona come una terapia! Per dire, adesso che studio sto sentendo i Die Antwoord e paradossalmente mi triggerano l'ASMR tanto quanto altri video! La musica elettronica mi rilassa tantissimo,le casse,il ritmo,la "texture" della musica... (intervento su forum asmritalia.blogfree.net, 27/5/20I2).

Siamo estremamente compatibili come carattere, interessi, passioni ecc... Ora, come mio solito, un livello di intimità alto "triggera" l'attrazione fisica, per cui sto avendo delle serie pulsioni verso questo ragazzo (intervento sul forum nienteansia.it, $15 / \mathrm{I} / 2018)$.

Nel primo caso il parlante fa riferimento alla sensazione di rilassamento (reazione detta "ASMR", "Autonomous Sensory Meridian Response") che può essere indotta da certi suoni; nel secondo si parla dell'eccitazione erotica "attivata" da una situazione di complicità. In entrambi gli esempi il triggerato sperimenta qualcosa di piacevole.

\section{C'è triggerare e triggerare...}

Gli esempi che abbiamo riportato sono tutti tratti da forum, community e social network: spesso attestano usi di nicchia, invalsi all'interno di comunità linguistiche a volte ristrette, non necessariamente comprensibili alla maggior parte dei parlanti. Alcuni video su Youtube sembrano testimoniare anche la popolarità di triggerare tra i giovani e i giovanissimi: lo troviamo, per esempio, in questa parodia adolescenziale di Volare di Fabio Rovazzi e Gianni Morandi (intitolata, appunto, Triggerare), in un video in cui un giovane giocatore commenta la sua partita a un videogioco ("Rekins mi triggera sbagliando il mio nome"), in un altro video in cui un giovane youtuber critica scherzosamente un altro canale ("Kyrenis mi triggeri duro"). Sono tutti esempi, questi e i precedenti, che suggeriscono come il triggerare segnalato dal nostro lettore tenda a inserirsi nel contesto di linguaggi di comunità online i cui tratti si discostano, in modo più o meno intenzionale, dallo standard.

Ciononostante, già una generica ricerca di triggerare sulle pagine italiane di Google restituisce risultati piuttosto consistenti per un termine che si presume usato principalmente in contesti di nicchia (II.ooo risultati circa solo per la forma all'infinito, ben 75.0oo per "triggera" - di cui 7600 per "mi triggera", II.ooo per "triggerato", 5000 per "ha triggerato", 700 per "ho triggerato" [dati raccolti nel marzo 2019]). Anche i risultati della ricerca sulle pagine italiane di Google Libri, per quanto contenuti nel numero (IIo occorrenze) sembrano documentare l'uso di un termine che ha un qualche impatto al di fuori degli ambiti specifici segnalati dal nostro lettore. $\grave{E}$ sufficiente, tuttavia, uno sguardo alle prime pagine prodotte dal motore di ricerca per capire come questi risultati diano voce a una varietà di usi e significati diversi di triggerare, tutti riconducibili all'idea dell'"innescare" ma non sempre corrispondenti a quelli che ci hanno interessato finora. Se, da una parte, questi dati possono ingannarci sul reale radicamento del nostro triggerare 'far arrabbiare', dall'altra il "rumore" che evidentemente li sporca ci suggerisce di indagare ulteriormente la questione.

Da un esame di Google Trends ci appare subito chiaro come, per lo meno dal punto di vista morfologico, triggerare non sia una formazione recentissima, dato il suo ricorrere con costanza nelle ricerche degli utenti italiani a partire almeno dal 2004 (anno a partire dal quale la piattaforma permette di fare ricerche). 
Il grafico che riproduciamo sotto ci mostra alcuni picchi di interesse intorno al $2004 \mathrm{e}$ al $2006 \mathrm{e} \mathrm{un} \mathrm{più} \mathrm{costante,}$ seppur contenuto, rialzo a partire dal 2017. Gli argomenti che appaiono correlati a queste ricerche rimandano a interessi quali l'elettronica e l'informatica.

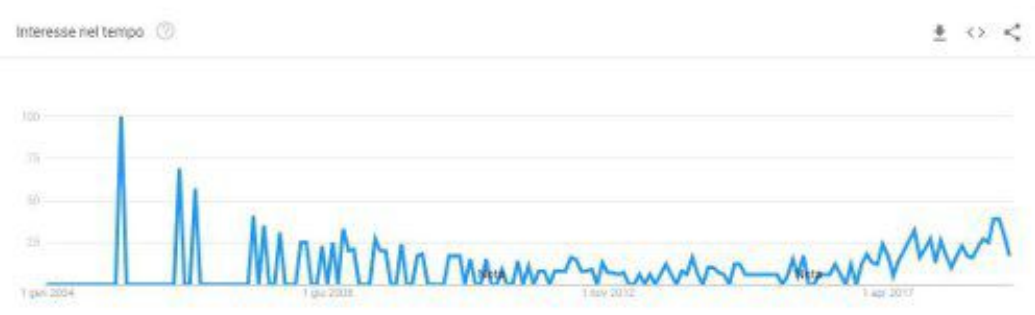

[L'andamento percentuale delle ricerche di triggerare sulle pagine in italiano di Google dal 2004 a oggi]

Ricordiamo che l'andamento descritto dai grafici di Google Trends non riporta il numero effettivo delle ricerche, ma, in percentuali relative, gli aumenti di interesse relativi a determinati argomenti. Per triggerato, invece, la presenza nelle ricerche appare meno consistente, con alcune impennate periodiche e una presenza più regolare dal 2017 in poi.

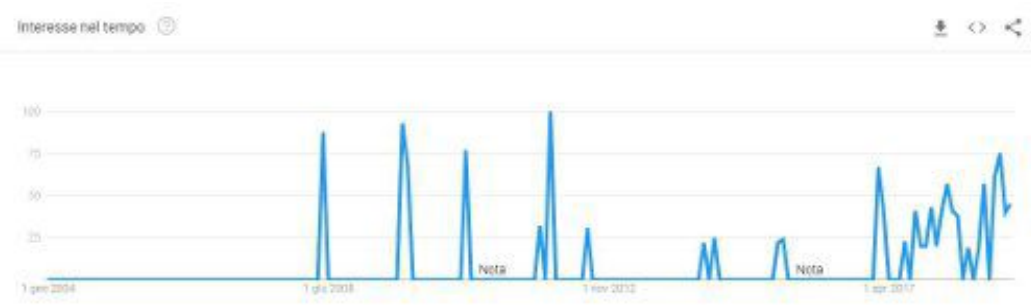

[L'andamento percentuale delle ricerche di triggerato sulle pagine in italiano di Google dal 2004 a oggi]

I dati relativi alla corrispondente forma inglese, triggered (participio passato di [to] trigger), possono aiutarci nell'interpretazione dei risultati precedenti. Anche in questo caso si registra un boom a partire dal 2017 tanto nelle ricerche italiane (come esemplifica il grafico qui sotto), tanto in quelle mondiali. La differenza significativa rispetto alle ricerche sulle pagine italiane delle forme adattate è che in questo caso il picco di attenzione è preceduto da un interesse che sembra, in percentuale, meno significativo negli anni precedenti.

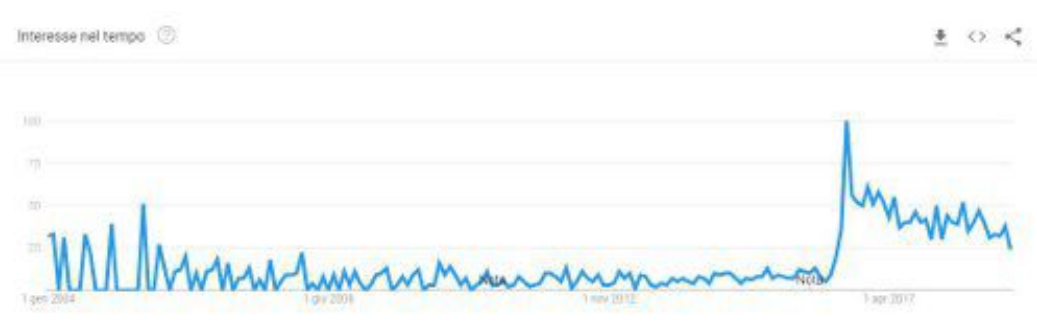

[Le ricerche di triggered sulle pagine in italiano di Google dal 2004 a oggi]

Confrontando i dati dei tre grafici, si ha innanzitutto l'impressione che per molti utenti di Google.it e Google.com (si presume, dunque, tendenzialmente italofoni i primi e anglofoni i secondi) le forme triggered e triggerato siano diventate d'improvviso più interessanti intorno al 2016/17. Google Trends, che riporta anche una serie di termini a cui le ricerche sarebbero correlate, ci aiuta a capire perché, suggerendoci sia per l'italiano che per l'inglese l'associazione con la parola meme. Appare naturale, a questo punto, far corrispondere l'aumento di interesse per la parola al momento di massima viralità del meme intitolato proprio "Triggered": un "tormentone" web che, come accuratamente riporta il sito Know your meme, si origina intorno al $2012 \mathrm{e}$ si diffonde, per lo meno nel mondo anglofono, intorno al 2015/2016. "Triggered" si propaga, come normalmente succede con i meme (o memi), in moltissime forme diverse, tutte variazioni di una forma originale, apprendiamo ancora - e con dispiacere - da Know your meme, nata per deridere una persona specifica e con intenti antifemministi. Col tempo e nei passaggi da una variante all'altra, perso il riferimento originario, i meme riconducibili al modello "Triggered" hanno continuato a ironizzare sulla capacità di certi oggetti, avvenimenti, 
parole di urtare la sensibilità e provocare la rabbia: diremmo appunto, adattando la forma inglese all'italiano, di triggerare qualcuno.

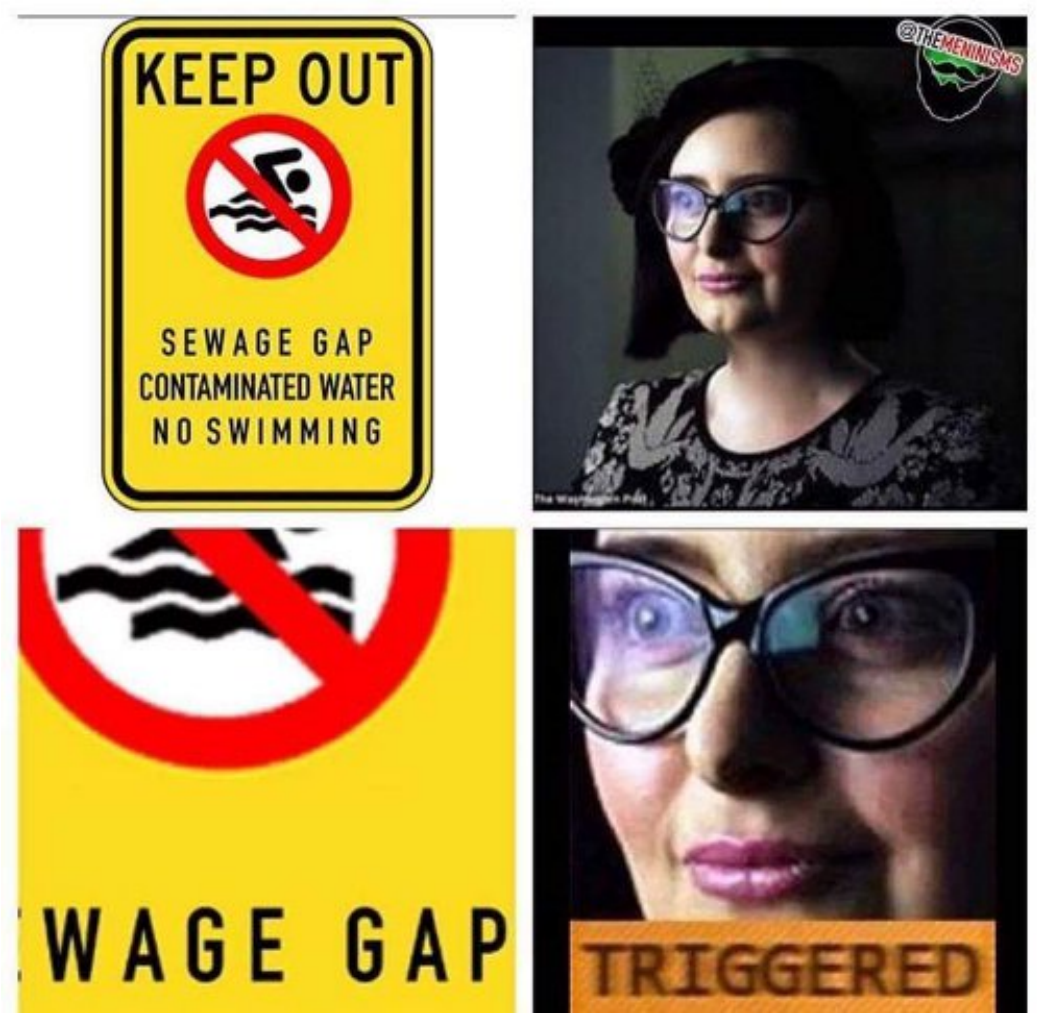

[Esempio di un meme "Triggered"]

I grafici, tuttavia, ci dicono anche che il verbo triggerare già incuriosiva gli italofoni prima che il successo del meme lo rendesse popolare. Se la forma che interessa al nostro lettore, infatti, usata negli ultimi anni col significato specifico che abbiamo visto, è probabilmente un adattamento creatosi sull'onda della diffusione - in origine anglofona e successivamente approdata sul web italiano - di certi contenuti virali, più ampio è il discorso riguardante la presenza generale del verbo triggerare in italiano. I dati forniti da Trends suggeriscono ciò che i risultati di ulteriori indagini confermeranno fra poco, e che risponde alla domanda del nostro lettore: triggerare può essere considerato una parola nuova, ma soltanto dal punto di vista semantico. Questo significa che la forma triggerare già esisteva in italiano con altri significati, e che, negli ultimi anni, essa ha assunto un significato nuovo (quello di cui si è parlato finora, che abbiamo comunque visto essere molto plastico e vago). Per capire meglio gli altri usi di triggerare, quelli di origine meno recente, è utile dare prima uno sguardo all'inglese.

\section{In inglese: triggered e to trigger}

È interessante notare come anche in contesti anglofoni l'uso di triggered susciti attenzione e curiosità tanto quanto, se non di più, quello del suo adattamento italiano: anche in questo caso il suo boom di popolarità, da ricondurre al diffondersi del meme, sembra attualmente legato ai linguaggi delle comunità online, ma è comunque abbastanza forte da determinare l'insorgere della percezione che la parola sia un neologismo. Non è raro, navigando sulle pagine in inglese di Google, imbattersi in siti che parlano di triggered come di una parola nuova, o comunque improvvisamente diventata popolare. Lo testimoniano articoli come questo del febbraio 2017 (Matt Daniels, Where Slang Comes From, pudding.cool, 2017) o questo del maggio dello stesso anno (Bri Di Monda, Let's discuss the ethymology of the word "triggered", www.adolescent.net, I/5/2017), nei quali gli autori registrano l'esplosione di popolarità del termine e ne indagano le modalità, le cause o la storia. Triggered è inoltre registrata su Urban Dictionary dall'aprile 2015 e, dal dicembre 2017, sul database Neologisms "autogestito" dagli studenti della Rice University di Houston. Il significato che queste schede gli associano è simile a quello di cui abbiamo parlato per l'italiano: si dice triggered chi è urtato da qualcosa che ha innescato la memoria di un'esperienza passata dolorosa, irritante o spiacevole ("getting filled with hate after seeing, hearing or experiencing something you can't stand", da Urban Dictionary). 
Come spiegano questi articoli, anche in inglese il verbo to trigger era già usato ben prima del successo del meme (l'OED ne documenta l'uso almeno dagli anni 'zo del Novecento) sia nel senso letterale di 'rilasciare il grilletto di una pistola o altro strumento simile', sia in quello esteso e più generico di 'innescare', 'causare un altro evento, come per esempio in una reazione a catena', 'scatenare', 'attivare'. Precedente al meme ed esteso sembra essere il suo impiego in elettronica, per esempio, dove si usa sia transitivamente nel senso di 'innescare un cambio di comportamento di una periferica', sia in intransitivamente con riferimento alla periferica stessa, nel senso di 'cambiare stato in relazione a un segnale ricevuto'.

Di uso comune è anche il nome trigger 'grilletto', attestato in inglese fin dal Seicento e attualmente impiegato anche nel senso figurato di 'causa scatenante'. Tra i molti nomi composti in cui è presente segnaliamo trauma trigger, impiegato in ambito medico e psicologico per identificare oggetti ed esperienze in grado di innescare, appunto, il ricordo di traumi subiti in passato (per esempio, i colpi di pistola per gli ex soldati affetti da disturbo da stress post-traumatico). Il sintagma sembra piuttosto diffuso: 26.000 circa sono i risultati per una ricerca virgolettata di "trauma trigger" sulle pagine inglesi di Google, I8oo su quelle di Google Books. I risultati partono dal 200I (zoro su libri, siti e riviste specialistiche) e cominciano a diventare consistenti intorno al 20I2, crescendo esponenzialmente. È interessante notare come l'espressione sia usata anche al di fuori dell'ambito specialistico da cui proviene: molte delle pagine restituite dal motore di ricerca contengono articoli di argomento psicologico ma di taglio divulgativo. Il fatto che il concetto di "trigger psicologico" sia a disposizione anche dei parlanti non specialisti è confermato dall'enorme diffusione dell'espressione "trigger warning" (circa 4.900.00o risultati su Google.com), che indica un particolare tipo di premessa a testi, video, immagini: quello che avverte, appunto, che il loro contenuto potrebbe urtare la sensibilità di qualcuno.

\section{In italiano: trigger...}

Tornando ai corrispondenti italiani, notiamo una situazione solo in parte simile. L'uso del sostantivo trigger è registrato da alcuni vocabolari (Zingarelli, GRADIT, Vocabolario Treccani online) come tecnicismo: tuttavia, a differenza che in inglese, il suo impiego in psicologia non è segnalato. Questo non significa che il termine non sia d'uso comune in quel particolare contesto tecnico (cosa che invece è ragionevole presumere, per di più considerato che la lingua veicolare della ricerca accademica è l'inglese), ma semmai che questo suo impiego non sia (ancora?) così diffuso tra i non specialisti da determinarne la segnalazione da parte della lessicografia.

Lo Zingarelli, che lo registra a partire dall'edizione 2017, definisce per esempio trigger in relazione all'uso che se ne fa nell'ambito della tecnologia come 'dispositivo che si attiva automaticamente al verificarsi di certe condizioni', dell'economia come 'valore limite (di un prezzo, di un titolo, ecc.) al raggiungimento del quale scattano procedure di salvaguardia' e della medicina come 'area di ipereccitabilità di un organo la cui stimolazione determina una reazione istantanea. Cercando di semplificare, al di là dei significati di volta in volta specifici che assume nei vari contesti, un trigger è qualcosa che dà l'innesco a un processo. La prima attestazione è fatta risalire al 195 I.

I risultati della ricerca sugli archivi storici di alcuni quotidiani ("La Stampa": 287 risultati; "La Repubblica": 157 risultati; "Il Corriere della Sera": 94 risultati) testimoniano un uso giornalistico di trigger iniziato negli anni '7o (quando compare l'espressione trigger-price in un articolo del 1978) e divenuto piuttosto consistente nei 'go, quasi sempre all'interno di articoli riferiti ad argomenti tecnici. I contesti d'uso sono disparati: si va dall'elettronica, alla fisica, alla medicina, alla dietologia e alla psicologia, all'economia, alla musica, per citarne alcuni. Il termine compare negli usi più datati tendenzialmente all'interno di composti:

Ad esempio, la produzione è diminuita nella Cee e in Giappone, è cresciuta invece negli Stati Uniti grazie ad un'apprezzabile ripresa del mercato interno e alle possibilità di contenere le importazioni con l'applicazione del "trigger-price" (Renzo Villare, I giganti Usa accusano la Cee di esportare acciaio sottocosto, "La Stampa", 3/10/1978).

A sua volta questa escalation si sarebbe automaticamente tradotta in un trigger-wire, il grilletto di un confronto generalizzato a livello di armi strategiche Usa-Urss (Vladimiro Odinzov, L'anno zero della sicurezza, "La Repubblica", 18/4/1987).

Secondo il dottor Stephen Gullo, ci sono alcuni alimenti, definiti trigger food, letteralmente 'cibi-grilletto', capaci di sviluppare in modo particolare il nostro appetito (Francesco Collenghi, La retro-dieta, "La Repubblica", 19/9/1995).

Più recentemente, invece, anche da solo: 
Dal manubrio un comando ergonomico integrato consente di selezionare il menu desiderato e due trigger di selezione permettono di personalizzare il display dello schermo [...] (Eicma 20I8, Peugeot all'attacco, "La Repubblica", 5/ıo/20I8).

Poiché quasi il 40\% della popolazione è geneticamente predisposta alla celiachia, gli autori ritengono che identificare dei trigger ambientali possa essere importante (Irma D'Aria, Celiachia, un virus 'accende' la malattia nei bambini predisposti, "La Repubblica", I8/2/2019).

Una massiccia presenza nella rete (II.500.0oo risultati sulle pagine in italiano di Google, 25.200 sulle pagine italiane di Google Libri) fuga ogni dubbio riguardo all'effettivo radicamento di questo prestito non adattato. Riguardo ai campi di impiego di trigger, i risultati della ricerca sulla rete sembrano confermare quanto è emerso dagli archivi dei quotidiani: la parola è usata in ambiti svariati, tecnico-specialistici (elettronica, musica, informatica, medicina, psicologia, linguistica ecc.), spesso per nominare un "attivatore", vale a dire qualcosa in grado di dare avvio a un processo, o scatenare una qualche conseguenza. Il Vocabolario Treccani online cita addirittura trigger all'interno del lemma grilletto, spiegando come, in elettronica, quest'ultimo sia a volte impiegato per rendere il corrispondente inglese e indicare il 'congegno che porta un segnale di attivamento o di comando' - testimoniando cosi, indirettamente, il fatto che in quest'ambito l'anglismo ha maggiore diffusione del traducente.

\section{... e triggerare}

La presenza solida della base non adattata trigger ha evidentemente reso naturale in italiano la formazione di triggerare. Anche se i vocabolari non la registrano, la parola sembra essere in uso da alcuni decenni, e dunque ben da prima che il diffondersi del meme determinasse la creazione di un triggerare come forma adattata. La prima impressione ricavata da una ricerca sulle pagine italiane di Google per gli anni precedenti al 2015 è quella di un verbo usato principalmente in elettronica, in informatica e in ambito musicale. Nei primi due ambiti triggerare corrisponde grosso modo ai più familiari 'far scattare', 'innescare', o, in certi casi, 'sincronizzare'. Attraverso determinate apparecchiature, come l'oscilloscopio, si triggerano segnali; oltre a quello specialistico, sempre nel campo dell'elettronica il termine ha anche usi più generici: per esempio, c'è chi triggera la propria macchina fotografica. Durante l'attività di programmazione a essere triggerati sono processi e funzioni. Da qui al tecnicismo di ambito musicale il passo non è troppo lungo: triggerare uno strumento, nello specifico una batteria, significa rendere il suo suono campionabile sistemando sulle pelli specifici sensori. Di batterie, pad, piatti, rullanti triggerati si parla, naturamente, in relazione alla musica elettronica, e con una frequenza tale che Google.it, se interrogato in proposito, produce dati piuttosto consistenti (9990 risultati per "triggerate una batteria", 324 per "triggerare la batteria", 398 per "batteria triggerata", I48 per "triggerare il rullante").

L'uso di triggerare, inoltre, sembra diffuso da tempo in molti settori della ricerca accademica, specialmente in quelli scientifici (tradizionalmente più permeabili alla penetrazione di anglismi), nei quali il suo impiego è stato certamente incoraggiato da quello inglese del corrispondente to trigger. Ce ne rendiamo conto, per esempio, cercando su Google, Google Scholar e Google Libri, dove troviamo traccia di triggerare in testi editi a partire dagli anni Settanta: citiamo il primo, gli Atti del Congresso annuale dell'AICA (Associazione Italiana per il Calcolo Automatico, Bari, Edizioni Dedalo, p. 634) del 1976, nei quali si triggera un cursore; il secondo, l'Enciclopedia medica italiana del 1998 (2. Aggiornamento della seconda edizione, a cura di Luciano Vella, Firenze, USES, I998, p. I449), in cui triggerare è usato in riferimento alla tecnica di acquisizione di impulsi nelle apparecchiature per la Risonanza Magnetica Nucleare, e infine l'Atlante di Anestesia e Terapia intensiva (di Lothar Ullrich, Dietmar Stolecki, Matthias Grünewald, Antonio Delfino Editore, 2009, p. I23), in cui di triggerare si testimonia l'uso intransitivo: è il paziente che triggera, nel senso che inizia un atto inspiratorio da solo, senza l'ausilio del macchinario per la ventilazione artificiale. Più recente, ma in grado di documentare anche l'uso transitivo nello stesso contesto, è una pubblicazione edita da Springer nel 20Io, in cui si triggera lo stesso apparecchio per la ventilazione artificiale (Stefano Nava, Francesco Fanfulla, Ventilazione meccanica non invasiva, Springer, Milano 20I0, p. 29). Il verbo ricorre anche nelle tesi di laurea: ecco per esempio la dissertazione di uno studente di Fisica dell'a.a. 2orı-20I2, in cui si triggera la scarica di un diodo, e quella, dello stesso anno, di uno studente di Ingegneria, dove a essere triggerato è "un evento di handover".

È evidente come il significato che il verbo assume in queste pubblicazioni sia di volta in volta strettamente specialistico e resti piuttosto lontano da quello, di registro completamente diverso, che interessa al nostro lettore. Tuttavia, è da notare come anche all'interno dei contesti specialistici triggerare sia talvolta impiegato in senso più vago: lo vediamo in questo articolo in cui si parla di terremoti, dove lo specialista intervistato spiega 
come certe attività ingegneristiche possano "alterare lo stato meccanico della crosta terrestre in maniera sufficiente da triggerare terremoti", usando triggerare nel senso di - citiamo le sue parole - "anticipare un terremoto che senza l'intervento umano sarebbe avvenuto più tardi"; e lo vediamo anche in questa tesi di laurea in Architettura (a.a. 2014-2015), il cui obiettivo è quello di "triggerare la ricerca" su Haiti, cioè semplicemente di 'darle avvio'.

In questi ultimi casi, in cui l'impiego di triggerare non pare legato alla specificità tecnica dell'attività descritta, il verbo sembra sostituibile con alternative italiane in modo del tutto indolore. L'opinione è condivisa anche da alcuni "addetti ai lavori": già nel 1996 Tullio Regge (significativamente intervistato insieme all'allora presidente dell'Accademia della Crusca Giovanni Nencioni) dichiarava alla "Stampa" la propria insofferenza nei confronti dell'abuso di triggerare nel linguaggio della fisica:

Trigger in americano indica l'innesco di una bomba, è ciò che dà inizio a un processo. Non possiamo dire "innescare"? Basta cercare nellitaliano, le parole ci sono, ma nessuno vuole più fare fatica (Scienziati, fermatevi, state uccidendo litaliano, "La Stampa", 4/1/1996, p. I4).

Più di recente, il nostro verbo è stato citato da Margherita Hack, che lo sceglie addirittura come modello esemplare del lessico di una cattiva scrittura accademica:

Oggi questo problema [quello della difficoltà a esercitare la scrittura accademica da parte degli studenti, nota nostra] è più grave perché chi fa studi scientifici non ha molte occasioni di scrivere, se non quando si cimenta con la tesi. Quando leggo le tesi dei laureandi me ne rendo conto e noto fenomeni quali la scorretta sillabazione delle parole [...] o il ricorso sempre maggiore a parole straniere anche quando esiste il corrispettivo italiano. Spesso ciò crea risultati un po' buffi, come fittare (accordarsi) o triggerare (innescare) (Margherita Hack, Qualcosa di inaspettato, Laterza, Roma-Bari 2007).

Le perplessità che in passato il diffondersi di triggerare nel senso generico di 'innescare, dare avvio' ha suscitato in contesti formali come quello accademico non possono, tuttavia, essere riproposte tout court per il triggerare odierno: una parola che in pochi decenni, come si è visto, ha vissuto in molti contesti e registri, subendo processi di risemantizzazione e rilanciandosi infine in una veste nuova e in usi di nicchia, segnati oggi dalle forti dinamiche identitarie dei gruppi dei parlanti. Per gli usi segnalati dal nostro lettore, per esempio, la ricerca di un traducente appare certamente meno appropriata. Per di più anche il futuro di triggerare (per esempio, la sua possibilità di essere registrata nei vocabolari) è ancora molto incerto, dato che l'ambito d'uso al quale il nostro lettore fa riferimento, quello del web, è un campo dove è frequente veder diffondersi parole e modi di dire in modo improvviso e capillare, ma altrettanto, a volte, effimero. Non resta che aspettare, continuare a monitorare la nostra parola, e vedere dove la porterà l'uso.

\section{Cita come:}

Simona Cresti, Triggerare, "Italiano digitale”, 2019, VIII, 2019/1 (gennaio-marzo) , pp. 69-76. DOI: $10.35948 / 2532-9006 / 2019.3133$

\section{Copyright 2019 Accademia della Crusca}

Pubblicato con licenza creative commons CC BY-NC-ND 